\title{
Gödel logics with an operator shifting truth values
}

\author{
Matthias Baaz and Oliver Fasching* \\ Vienna University of Technology \\ Vienna, Austria \\ \{baaz,fasching\}@logic.at
}

\begin{abstract}
We consider GÃúdel logics extended by an operator whose semantics is given by $\mathfrak{I}(o(A))=\min \{1, r+\mathfrak{I}(A)\}$.
\end{abstract}

The language of propositional GÃúdel logics $\mathcal{L}^{\text {p }}$ consists of a countably infinite set Var of propositional variables and the connectives $\perp, \supset, \wedge, \vee$ with their usual arities. We will consider extensions by a unary connective $o$, by a unary connective $\triangle$ or by both. For any $r \in[0,1]$, a GÃúdel $r$-interpretation $\mathfrak{I}$ maps formulas to $V$ such that $\mathfrak{I}(\perp)=0$,

$$
\begin{aligned}
& \mathfrak{I}(A \wedge B)=\min \{\mathfrak{I}(A), \mathfrak{I}(B)\}, \\
& \mathfrak{I}(A \vee B)=\max \{\mathfrak{I}(A), \mathfrak{I}(B)\}, \\
& \mathfrak{I}(A \supset B)= \begin{cases}1 & \mathfrak{I}(A) \leq \mathfrak{I}(B), \\
\mathfrak{I}(B) & \mathfrak{I}(A)>\mathfrak{I}(B) .\end{cases}
\end{aligned}
$$

If the language contains $o$ resp. $\triangle$, we additionally require

$$
\begin{gathered}
\mathfrak{I}(o(A))=\min \{1, r+\mathfrak{I}(A)\}, \\
\mathfrak{I}(\triangle(A))= \begin{cases}1 & \mathfrak{I}(A)=1 \\
0 & \mathfrak{I}(A)<1 .\end{cases}
\end{gathered}
$$

Let $G$ be some Hilbert-Frege style proof calculus that is sound and complete for propositional GÃúdel logics (without $o$ and $\triangle$ ), e.g. take a proof system for intuitionistic logic, plus the schema of linearity $(A \supset B) \vee(B \supset A)$, see [3] or, alternatively, use one of the systems described in [4]. We prove that $G$ enhanced by the axiom schemata $(\perp \prec o \perp) \supset(A \prec o A)$, $(\perp \leftrightarrow o \perp) \supset(A \leftrightarrow o A)$, and $o(A \supset B) \leftrightarrow(o A \supset o B)$ is sound and complete w.r.t. the above semantics. Generalizing ideas from [2], we also give an algorithm that constructs a proof for any valid formula. However, this semantics fails to have a compact entailment.

The above proof system can also be further combined with a proof system for $\triangle$, see [1], to yield a sound and complete calculus for the valid formulas in that language.

While the propositional fragment has quite a simple structure, we will show that first order GÃúdel logic enhanced by this ring operator is not recursively enumerable, using a technique by Scarpellini [5] employed for tukasiewicz logic. This ring operator makes the borderline of similarities and contrasts between Łukasiewicz logic visible.

The situation changes if one interprets $o$, more generally, as a function with certain monotonicity properties.

* partially supported by Austrian Science Fund (FWF-P22416) 


\section{References}

[1] M. Baaz. Infinite-valued GÃüdel logics with 0-1-projections and relativizations. GÃúdel '96, Lecture Notes Logic 6:23-33. 1996.

[2] M. Baaz, H. Veith. Interpolation in fuzzy logic. Arch. Math. Logic 38:461-489. 1999.

[3] M. Dummett. A Propositional Calculus with Denumerable Matrix. J. Symb. Log. 24(2):97-106. 1959.

[4] P. HÃajek. Metamathematics of fuzzy logic. Trends in Logic - Studia Logica Library. Kluwer. 1998.

[5] B. Scarpellini, Die Nichtaxiomatisierbarkeit des unendlichwertigen Pradikatenkalk $\tilde{A}$ ijls von Eukasiewicz. J. Symb. Log. 27:159-170. 1962. 